Carbon nanotube composite coating of neural microelectrodes preferentially improves the multiunit signal-to-noise ratio

This content has been downloaded from IOPscience. Please scroll down to see the full text.

2011 J. Neural Eng. 8066013

(http://iopscience.iop.org/1741-2552/8/6/066013)

View the table of contents for this issue, or go to the journal homepage for more

Download details:

IP Address: 193.205.81.18

This content was downloaded on 24/11/2016 at 09:59

Please note that terms and conditions apply.

You may also be interested in:

Quantifying long-term microelectrode array functionality using chronic in vivo impedance testing

Abhishek Prasad and Justin C Sanchez

Evaluation of PEDOT films for improving neural recordings

Kip A Ludwig, Jeffrey D Uram, Junyan Yang et al.

Progress towards biocompatible intracortical microelectrodes for neural interfacing applications Mehdi Jorfi, John L Skousen, Christoph Weder et al.

Conducting polymer coated neural recording electrodes

Alexander R Harris, Simeon J Morgan, Jun Chen et al.

Integrated device for optical stimulation and spatiotemporal electrical recording of neural

activity

Jiayi Zhang, Farah Laiwalla, Jennifer A Kim et al.

A MEMS-based flexible multichannel ECoG-electrode array

Birthe Rubehn, Conrado Bosman, Robert Oostenveld et al.

A new EC--PC threshold estimation method for in vivo neural spike detection

Zhi Yang, Wentai Liu, Mohammad Reza Keshtkaran et al. 


\title{
Carbon nanotube composite coating of neural microelectrodes preferentially improves the multiunit signal-to-noise ratio
}

\author{
Gytis Baranauskas $^{1,3}$, Emma Maggiolini ${ }^{1,3}$, Elisa Castagnola ${ }^{1,3}$, \\ Alberto Ansaldo ${ }^{1}$, Alberto Mazzoni ${ }^{1}$, Gian Nicola Angotzi ${ }^{1}$, \\ Alessandro Vato ${ }^{1}$, Davide Ricci ${ }^{1}$, Stefano Panzeri ${ }^{1}$ and \\ Luciano Fadiga ${ }^{1,2,4}$ \\ ${ }^{1}$ Robotics, Brain and Cognitive Sciences Department, Italian Institute of Technology, Genoa, Italy \\ ${ }^{2}$ Section of Human Physiology, University of Ferrara, Ferrara, Italy \\ E-mail: luciano.fadiga@iit.it
}

Received 22 July 2011

Accepted for publication 13 October 2011

Published 8 November 2011

Online at stacks.iop.org/JNE/8/066013

\begin{abstract}
Extracellular metal microelectrodes are widely used to record single neuron activity in vivo. However, their signal-to-noise ratio (SNR) is often far from optimal due to their high impedance value. It has been recently reported that carbon nanotube (CNT) coatings may decrease microelectrode impedance, thus improving their performance. To tease out the different contributions to SNR of CNT-coated microelectrodes we carried out impedance and noise spectroscopy measurements of platinum/tungsten microelectrodes coated with a polypyrrole-CNT composite. Neuronal signals were recorded in vivo from rat cortex by employing tetrodes with two recording sites coated with polypyrrole-CNT and the remaining two left untreated. We found that polypyrrole-CNT coating significantly reduced the microelectrode impedance at all neuronal signal frequencies (from 1 to $10000 \mathrm{~Hz}$ ) and induced a significant improvement of the SNR, up to fourfold on average, in the $150-1500 \mathrm{~Hz}$ frequency range, largely corresponding to the multiunit frequency band. An equivalent circuit, previously proposed for porous conducting polymer coatings, reproduced the impedance spectra of our coated electrodes but could not explain the frequency dependence of SNR improvement following polypyrrole-CNT coating. This implies that neither the neural signal amplitude, as recorded by a CNT-coated metal microelectrode, nor noise can be fully described by the equivalent circuit model we used here and suggests that a more detailed approach may be needed to better understand the signal propagation at the electrode-solution interface. Finally, the presence of significant noise components that are neither thermal nor electronic makes it difficult to establish a direct relationship between the actual electrode noise and the impedance spectra.
\end{abstract}

S Online supplementary data available from stacks.iop.org/JNE/8/066013/mmedia

(Some figures in this article are in colour only in the electronic version)

\footnotetext{
3 These authors contributed equally to this work.

4 Author to whom any correspondence should be addressed.
} 


\section{Introduction}

Extracellular microelectrodes are one of the most important tools to study the function of the brain in vivo [1]. The neural signals recorded by extracellular microelectrodes have provided a significant amount of knowledge about brain function in behaving animals [2] and are central to a number of clinical applications [3], including the development of brain machine interfaces (BMIs), a technology that translates cortical brain activities into commands for operating robotic arms or other external devices [4]. Such neural signals are distributed over a wide range of frequencies and each part of the frequency spectrum reflects different neural processes. The low frequency band (approximately $1-250 \mathrm{~Hz}$ ) contains the so-called local field potentials (LFPs) and reflects synaptic activity and other subthreshold integrative processes [5]. Spikes of individual neurons can be detected in the high frequency range $(500-3000 \mathrm{~Hz})$ while the power variations in the same frequency band represent the overall spiking activity of multiple neurons close to the electrode tip (multiunit activity signal, MUA) [5-7]. Because of their different neural origins, different frequency bands of extracellularly recorded neural signals often carry complementary information about external functional correlates such as sensory activity or motor commands [8-11] and their simultaneous measure increases the amount of information available, e.g. to control prosthetic devices [12].

An ideal extracellular microelectrode should provide the highest signal quality, expressed as signal-to-noise ratio (SNR), for the whole frequency band containing any useful neural information. Currently, the vast majority of neurophysiology laboratories record extracellular neural signals using microelectrodes with metal tips [1,2]. However, their SNR is often far from ideal because of relatively large noise levels, mainly thought to arise from thermal noise, directly related to the microelectrode impedance values $[1,13,14]$. Thermal noise amplitude becomes larger when the electrode impedance real part increases. The electrode impedance real part is inversely proportional to the electrode tip surface area and large area electrodes should generate thermal noise of small amplitude. Theoretical and experimental considerations, however, indicate that to obtain high selectivity in action potential recordings from individual neurons, the microelectrode tip size in any direction should not exceed $20 \mu \mathrm{m}$, corresponding to the maximal unmodified surface area of $\sim 1000 \mu \mathrm{m}^{2}[1,15]$. Due to such a small exposed metal area, the microelectrode impedance is often very large $(\sim 0.1-1 \mathrm{M} \Omega)$ leading to high thermal noise levels and, consequently, low SNR.

To improve the performance of modern extracellular microelectrodes, various surface modifications that lower the tip impedance without significantly increasing the tip size have been studied [16-24]. A significant advance was recently made by Keefer and colleagues [21], who proposed using carbon nanotube (CNT) coatings to increase the tip surface area, thus decreasing the microelectrode impedance without significantly affecting the geometrical tip size. This study reported that coating microelectrodes with CNT composites improves neural signal quality for all three major types of extracellular microelectrode signals-LFPs, MUA and spikes [21]. The reported data demonstrate, however, that there is no straightforward relationship between the electrode impedance and its properties. Theory shows that the thermal noise root mean square (rms) should be proportional to the square root of the impedance real part [20,21,23]. Thus, for the observed drop in the impedance values of 30-100 times following CNT coating, the expected thermal rms noise reduction is more than fivefold or $>80 \%$. However, in practice CNT coating reduces the microelectrode rms noise only by 40 $60 \%$, i.e. approximately twofold, much less than predicted. This discrepancy between the decrease in the microelectrode impedance real part and the rms noise contradicts the view that the impedance determines most of the microelectrode performance $[1,14,16,20,25]$. Similarly, theory predicts that the neural signal amplitude is attenuated only when the amplifier input impedance is comparable to the microelectrode impedance in a given frequency range $[1,14,15]$. Since most modern amplifiers have input impedance of $30 \mathrm{M} \Omega$ or more, no significant changes in neural signal amplitude should occur for electrodes, the impedance of which is well below $10 \mathrm{M} \Omega$. However, the experimental results show that even in the high frequency range $(>500 \mathrm{~Hz})$, in which non-modified electrode impedance is below 2-3 $\mathrm{M} \Omega$, coating with an electrochemical co-deposition of CNTs and conductive polymers, typically polypyrrole (PPy), or gold, increases the neural signal amplitude [20,21].

These types of coatings were also tested by us [26] and we found that, compared to the electrodes coated with the goldCNT composite, those coated with PPy and CNT composite (PPy-CNT) have better electrochemical performances, in agreement with Keefer and colleagues [21]. Although the PPy-CNT coating can degrade within hours during constant polarization [27], we found that the electrode properties are essentially unchanged during several hour long acute recordings when no polarization is applied and the subsequent tests showed that the impedance is changed by less than $25 \%$ after such recording sessions.

Based on these results, to understand to what extent the impedance measurements can predict the microelectrode performance, we investigated in detail impedance, noise and neural signals on PPy and chemically-functionalized multi-walled CNT (MWCNT-PPy) composite-coated metal microelectrodes.

Our working hypothesis is that the main electrode noise component derives from sources other than the thermal noise. In contrast to thermal noise, other noise components are frequency dependent. Thus, to tease out the contribution of thermal noise and other noise sources to the overall microelectrode noise, we compared the impedance and noise spectra of MWCNT-PPy modified microelectrodes and non-coated, pristine, platinum/tungsten microelectrodes. Since both the power of neural signals and the increase in neural signal power produced by MWCNT-PPy coating are frequency dependent $[21,28]$, we also performed neural signal spectral analysis in order to compare noise and neural signal levels and, thus, to obtain SNR frequency dependence for the 
(A)

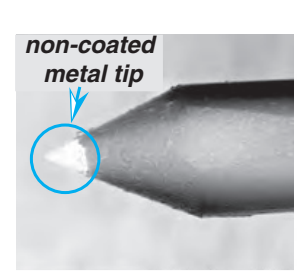

$50 \mu \mathrm{m}$ electrode surface

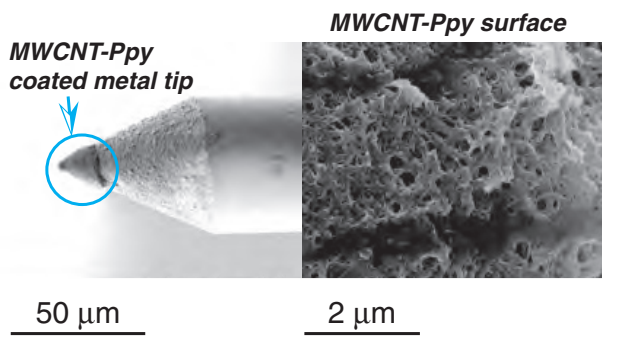

(B)

impedance

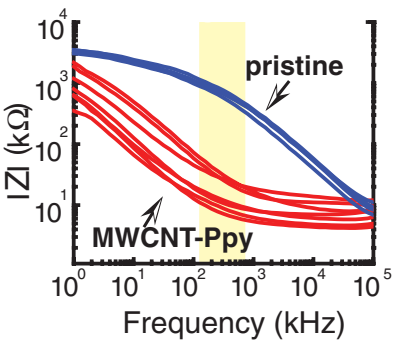

(C) phase

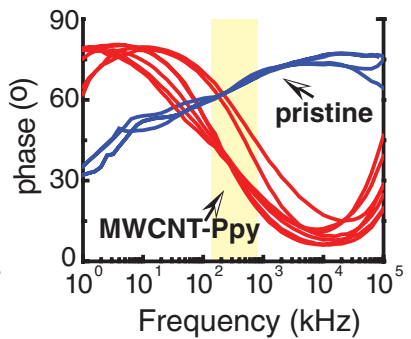

Figure 1. MWCNT-PPy coating strongly reduced the impedance of extracellular metal microelectrodes. (A) SEM images at low resolution show the tips of non-coated and coated microelectrodes. High resolution imaging reveals extensive porosity at sub-micron scale of MWCNT-PPy-coated microelectrodes. (B), (C) Impedance spectroscopy shows a significant decrease in the impedance modulus (B) and a complete change in the phase frequency dependence $(C)$. The light orange shaded area corresponds to the frequency range in which the background noise levels were reduced significantly after MWCNT-PPy coating.

whole neural signal frequency band. We found that, though the impedance spectroscopy can be used to predict some electrode properties, there is no direct relationship between the microelectrode performance and its impedance. This observation is explained in part by the presence of significant noise components with no direct relationship to the impedance values.

\section{Materials and methods}

\subsection{Metal microelectrodes}

Single core quartz insulated metal electrodes were prepared inhouse by mechanically grinding $95 \%$ platinum $/ 5 \%$ tungsten microwires of $20 \mu \mathrm{m}$ diameter coated with $30 \mu \mathrm{m}$ of quartz (ThomasRecording, Giessen, Germany). The impedance of these electrodes, measured in a saline solution at $1 \mathrm{kHz}$, was typically between 500 and $700 \mathrm{k} \Omega$ and the estimated exposed metal tip area was $\sim 900 \mu \mathrm{m}^{2}$ [26]. For neural activity measurements, two platinum/tungsten tetrodes (ThomasRecording, Giessen, Germany, 400-1200 k $\Omega$ at $1 \mathrm{kHz}$ ) were used. These specially designed microelectrodes have four independent recording sites of diameter between 14 and $26 \mu \mathrm{m}$, separated by less than $40 \mu \mathrm{m}$ on the tip of the same shaft (figure 6(B)). We have chosen such electrodes as they make it possible to record signals of the same neuron from more than one site [29]. In our experiments, two recording sites of the tetrode were coated with MWCNT-PPy while the other two remained untreated.

\subsection{PPy-CNT deposition}

We made a CNT electrochemical deposition with pyrrole in an aqueous solution using procedures recently described [21, 26, 30]. Briefly, PPy and chemically-functionalized multi-walled CNTs were co-electrodeposited from an aqueous solution of $0.5 \mathrm{M}$ pyrrole (Sigma-Aldrich), $1 \mathrm{mg} \mathrm{ml}^{-1} \mathrm{COOH}-$ MWCNTs (Nanocyl 3151, <4\% of -COOH functional groups) and $0.4 \%$ poly-sodium 4-styrene-sulfonate (PSS) (Sigma-Aldrich). As purchased, $\mathrm{COOH}-\mathrm{MWCNTs}$ were suspended in ultrapure water (Milli-Q, Millipore) by horn sonication $\left(6 \mathrm{~s}\right.$ at $66 \%$ duty cycle pulses, $4 \mathrm{~W} \mathrm{ml}^{-1}$ ) for minimum $15 \mathrm{~min}$ and up to $60 \mathrm{~min}$ while keeping the solution cooled with an ice bath. PSS and pyrrole were added to the suspension immediately afterwards and the solution was kept deoxygenated by bubbling with nitrogen. The electrochemical deposition was carried out under an inert atmosphere in potentiostatic mode. The polymerization potential was set to $550 \mathrm{mV}$ versus $\mathrm{Ag} / \mathrm{AgCl}$ reference electrode while the deposition time varied from 5 to $50 \mathrm{~s}$. The amount of charge passed during the deposition was between 100 and $400 \mathrm{mC}$ $\mathrm{cm}^{-2}$.

The result of electrochemical deposition was the formation of a nanostructured coating of PPy and MWCNTs on the microelectrode exposed tip (figure 1(A)). Reproducible coatings could be attained as demonstrated by the impedance modulus and phase measurements shown in figures 1(B) and $(\mathrm{C})$. Following preliminary tests, we selected deposition parameters in such a way that the microelectrodes used for this study had impedance at $1 \mathrm{kHz}$ no more than $25 \mathrm{k} \Omega$ and a total charge transfer capability no more than $200 \mathrm{mC} \mathrm{cm}^{-2}$.

\subsection{Electrochemical and morphological characterizations}

All measurements were carried out using a potentiostat/ galvanostat (Parstat 2273, Princeton Applied Research, Oak Ridge, TN, USA) and a standard three-electrode electrochemical cell configuration with a Pt wire as a counter electrode and the $\mathrm{Ag} / \mathrm{AgCl}$ reference electrode.

Galvano electrochemical impedance spectroscopy was performed in a saline solution $(0.9 \% \mathrm{NaCl})$ by applying a sine wave of $300 \mathrm{rms}$ nA current amplitude. The impedance values were determined at ten frequencies per decade over the range $1-10^{5} \mathrm{~Hz}$.

For cyclic voltammetry tests the potential on the working electrode was swept between 0.6 and $-1.0 \mathrm{~V}$ versus $\mathrm{Ag} / \mathrm{AgCl}$ in saline solution at a scan rate of $100 \mathrm{mV} \mathrm{s}^{-1}$, starting at open-circuit potential and sweeping in the positive direction first. The total charge transfer capability was calculated as the time integral of a whole cyclic voltammetry cycle.

The morphology of CNT nano-composites was examined using a cold field emission gun high resolution scanning electron microscope (HR-SEM, Jeol JSM-7500 FA) (figure 1(A)). 


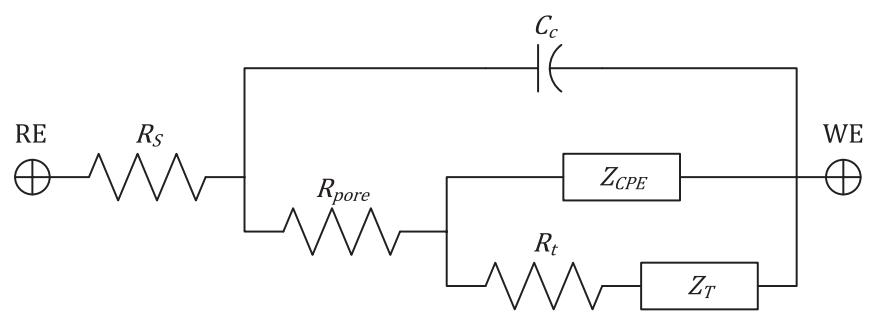

Figure 2. Equivalent circuit model of electrode-nanocoatingelectrolyte interface. WE stands for the working electrode and RE for the reference electrode. Role and equation of the single elements are reported in table 1.

\subsection{Fitting an equivalent circuit model to the impedance data}

To describe in detail the MWCNT-PPy-coated electrodeelectrolyte interface impedance, we used the equivalent circuit model (figure 2), proposed by Abidian and colleagues [31], that takes into account the conducting polymer coating interface, and the corresponding equations:

$$
Z=R_{s}+Z_{\text {elect }}=R_{s}+\frac{1}{\mathrm{j} \omega C_{c}+\frac{1}{R_{p}+\frac{1}{\frac{1}{Z_{\mathrm{CPE}}}+\frac{1}{R_{t}+Z_{T}}}}},
$$

where $R_{s}$ is the solution resistance, $Z_{\text {elect }}$ stands for the electrode impedance without the solution impedance, $j$ is the imaginary number and $\omega$ is the angular frequency, $C_{c}$ is the coating capacitance, $R_{p}$ the pore resistance, $Z_{\mathrm{CPE}}$ the interface capacitance, $R_{t}$ the charge transfer resistance and $Z_{T}$ the finite diffusion element.

To verify if the model satisfied the impedance response of our modified neural microelectrodes we estimated the parameter set of equation (1) by a best-fit procedure of the experimental impedance (both modulus and phase).

The best fit was achieved by minimizing (over the space of parameter values) the sum of square errors of model predictions of both modulus and phase. The minimization was performed using the Levenberg-Marquardt down-hill algorithm while constraining the parameters to remain in the real domain. We used as initial guess for the parameters the values for PPy nanotubes reported by [31], rescaled for the geometrical area of our electrode $\left(900 \mu \mathrm{m}^{2}\right)$. The statistical test of goodness of fit was assessed using the left $\chi^{2}$ test [32] which reports the $p$ value of the null hypothesis that data are generated by the model. We set the model acceptance threshold to $p>0.95$. We assessed the tolerance range for each parameter by varying its value around the best-fit value and computing the goodness-of-fit $\chi^{2} p$ value after each parameter variation. The tolerance range for each parameter was given by the range of variations for which the $\chi^{2}$ test significance remained above $p=0.95$. The narrower the tolerance range, the more accurate the estimation of the parameter value is. The wider the tolerance range, the less sensitive to the precise value of the parameter the fit between model and data is (figure 3).

\subsection{Animal surgery}

Experiments were carried out in acute sessions on eight anaesthetized Long-Evans male rats, weighing 300-400 g. Six animals were used for single electrodes and two for tetrodes. The experimental plan was designed in compliance with the Italian law regarding the care and use of experimental animals (DL116/92) and approved by the institutional review board of the University of Ferrara and by the Italian Ministry of Health. Rats were anaesthetized with a mixture of Zoletil $\left(30 \mathrm{mg} \mathrm{kg}^{-1}\right)$ and Xylazine $\left(5 \mathrm{mg} \mathrm{kg}^{-1}\right)$ delivered intraperitoneally. For the duration of the experiment, the depth of anaesthesia was monitored by testing for the absence of hindlimb withdrawal reflex and was maintained by additional doses of anaesthetic (i.p. or i.m.). Under anaesthesia, the body temperature was maintained at $36-38{ }^{\circ} \mathrm{C}$ with a thermostatically controlled heating pad. In each recording session the anaesthetized animal was placed in a stereotaxic apparatus (Myneurolab, St Louis, MO) and a small craniotomy $\left(2 \times 2 \mathrm{~mm}^{2}\right)$ was made in the parietal bone to expose the vibrissa region of the somatosensory cortex according to vascular landmarks and stereotaxic coordinates [33-35]. Dura mater was left intact and quartz-platinum/tungsten microelectrodes and tetrodes were lowered perpendicular through the cortical surface using a hydraulic microdrive (Kopf, 2650) to a depth of $>900 \mu \mathrm{m}$ (infragranular layer) [36-38]. After testing and confirming the placement of the electrodes by recording the extracellular neuronal discharges to manual whisker stimulation, the spontaneous activity of infragranular layer, consisting of bursts' firing (action potential clusters), was recorded [39]. Seven MWCNT-PPy-coated and five control, non-coated single electrodes were used in this study. Both modified and non-modified electrodes were tested on the same day, i.e. for each rat we tested at least one MWCNT-PPy-coated and one non-coated electrode. For each electrode one to three penetrations/tracks in a rat brain were made, in total seven tracks for non-coated electrodes and ten tracks for MWCNTPPy-coated electrodes. Three tracks were made with each of the two tetrodes. Each tetrode had two recording sites coated with MWCNT-PPy while the remaining two recording sites were left uncoated.

\subsection{Neural recordings and spike sorting}

The neural activity was recorded using a Plexon Multichannel Acquisition Processor (Plexon Inc. Dallas, TX, USA) 1000× preamplifier connected to a high impedance headstage $(\geqslant 40 \mathrm{M} \Omega, \mathrm{HST} / 16025-18 \mathrm{P}-\mathrm{GR})$ with the amplifier ground connected to the ground electrode. Most analyses were performed with custom written routines employing Igor Pro program (Wavemetrics, Lake Oswego, OR, USA) while spike sorting was performed with the Plexon offline sorter software.

For single unit separation (spike sorting), we employed the Plexon Expectation Maximization algorithm followed by manual verification of spike cluster quality. Our goal was to compare the quality of unit separation for different types of electrodes and not to achieve the best sorting results. We were more concerned to have a reproducible procedure and to have confirmation of the presence of a single unit in a cluster. 
(A)

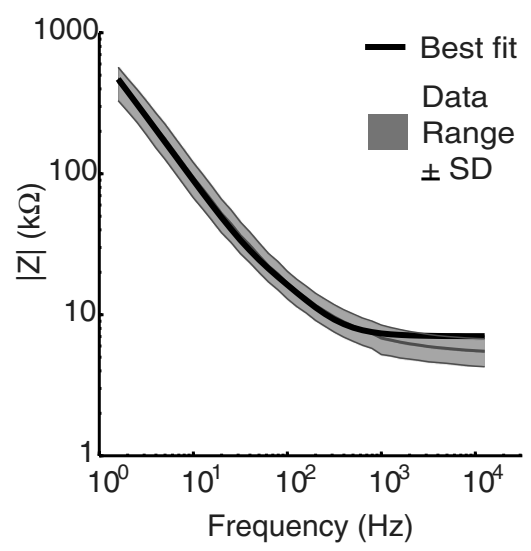

(B)

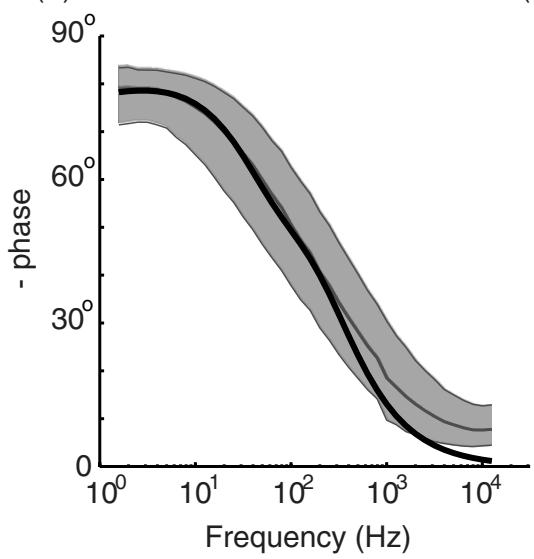

(C)

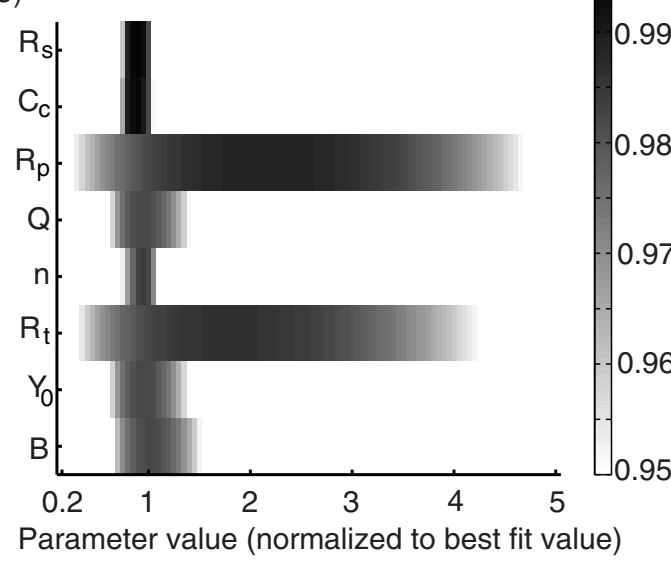

Figure 3. Measured impedance spectra and best model fit. (A) Impedance modulus. The grey area represents the mean \pm standard deviation range of the recorded data. The black line represents the best fit of the dataset obtained using the equivalent circuit model (figure 1, equation [1] in section 2). The grey line indicates the average impedance module across data. (B) Impedance phase. The fit was performed simultaneously for impedance phase and modulus and the best obtained fit with the parameter values of table 1 is shown (left $\chi^{2}$ test of goodness of fit; $p=0.985$ for the range $1 \mathrm{~Hz}-10 \mathrm{kHz}$ ). (C) Tolerance range for parameters estimated in the model. Parameters are normalized by dividing them by the corresponding best-fit values of table 1 . Each parameter was modulated in the range indicated on the $x$ axis while all the others were fixed to their best-fit value. The grey level bar indicates the level of significance of the $\chi^{2}$ goodness-of-fit test for the model for the given parameter value (up to a rejection threshold of $p<0.95$ indicated in white). The tolerance range for each parameter is indicated by the extent of the area around the reference value in which the $p$ value remains above $0.95 . R_{s}, C_{c}, R_{p}$ and $R_{t}$ definitions are provided in table 1. $Q$ and $n$ determine the behaviour of the interface capacitance $Z_{\mathrm{CPE}}$ and $Y_{0}$ and $B$ are the parameters in the equation for the diffusion element $Z_{T}$ (see the corresponding expressions in table 1).

A unit was confirmed only if

(i) a refractory period of $2 \mathrm{~ms}$ was present in the crosscorrelograms (the permitted rate was $<0.1 \%$ for $2 \mathrm{~ms}$ and $0 \%$ for $1 \mathrm{~ms}$ ) [11];

(ii) the number of events was $>500$;

(iii) ANOVA test showed that the quality of cluster separation was at $p<0.05$ significance level.

To estimate the background noise during bursts, we first built a histogram of signal distribution that was fit with a single Gaussian function $a \cdot \mathrm{e}^{-\frac{(x-b)^{2}}{2 c^{2}}}$. The parameter $c$ was assumed to correspond to rms noise.

\section{Results}

\subsection{Deposition and stability of microelectrodes}

Confirming previous results [20, 21, 26], MWCNT-PPy coating strongly reduced the average impedance modulus at $1 \mathrm{kHz}$ (the frequency most commonly used to characterize neural microelectrodes), from $354 \pm 22$ to $9.7 \pm 0.8 \mathrm{k} \Omega(p<$ $\left.5 \times 10^{-8}\right)$. At the same time, we have found that the average of the real part of the impedance, $Z_{\text {real }}$, which determines the thermal noise [20,40], is reduced from $56 \pm 5$ to 8.3 $\pm 0.2 \mathrm{k} \Omega$ in the $250-8000 \mathrm{~Hz}$ band, that is the frequency range used to detect MUA and spiking activity [5]. The impedance (measured by $\Omega$ mega-Tip-Z, WPI Inc., USA) changed by less than $25 \%$ following a full day recording session in the rat brain, suggesting that the MWCNT-PPy coating was reasonably stable.

\subsection{Validation of equivalent circuit model parameters}

The impedance spectra (figure 3 panels A and B) showed the typical behaviour for a high surface area electrode, with low frequency shifted pole and phase starting close to $-90^{\circ}$ and rising to zero with frequency. To fit the impedance data we employed a model proposed by Abidian and co-workers for PPy and poly(3,4-ethylenedioxytiophene) (PEDOT) nanotube coatings on neural microelectrodes (figure 2). The fit results presented in figure 3 show that this model reproduces most features of the impedance spectra of MWCNT-PPy-coated electrodes.

Parameter values reported in table 1 correspond to the best fit of experimental data $\left(\chi^{2}\right.$ test, $p=0.985$ for the range $1 \mathrm{~Hz}-10 \mathrm{kHz}$ ). The tolerance range of the obtained numerical values was estimated by modulating the value for each parameter and evaluating how much the goodness of the fit degraded when the value was moved progressively away from the best-fit value. Figure 3(C) shows the significance of the $\chi^{2}$ test for different values of each parameter (normalized by dividing by the best-fit value). For some parameters $\left(R_{S}\right.$ and $C_{c}$ ), the tolerance range (represented as gray horizontal bars in figure $3(\mathrm{C})$ ) is narrow, i.e. small variations are sufficient to bring the significance of the fit below the threshold value (set at $p=0.95$ ). This means that the model is very accurate in estimating these parameters. On the other hand, parameters such as $R_{p}$ and $R_{t}$ could be varied for as much as a factor of 4 and still give good agreement with the data. For these parameters, the model is not able to well estimate the parameter values. 


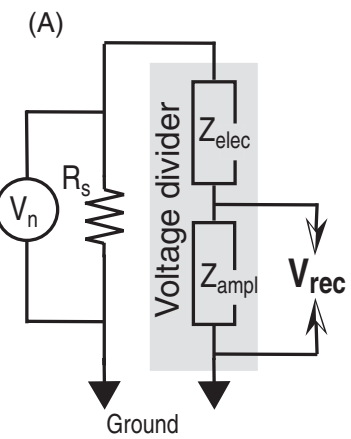

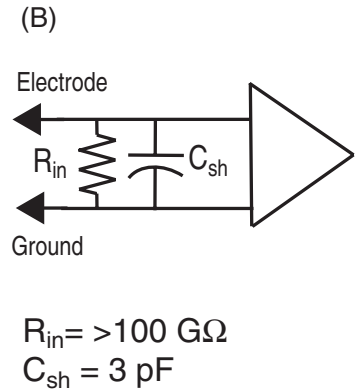

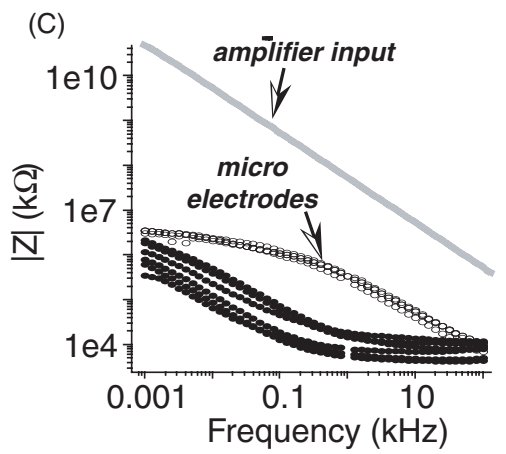

Figure 4. Equivalent input circuit model for the amplifier. (A) The configuration of the neural signal generator $\left(V_{n}\right)$ and the amplifier input signal $\left(V_{\text {rec }}\right)$ shown with respect to the electrode, solution and the amplifier input impedance. Arrows indicate places in the circuit, in which the voltage divider appears. (B) Amplifier input equivalent circuit. (C) The impedance modulus of the amplifier input (thick grey line), real (filled circles) and imaginary parts (open circles) of all used microelectrodes plotted against frequency. Note the difference of more than two orders between the impedance modulus of the amplifier input and microelectrodes.

Table 1. Parameter values obtained by fitting experimental impedance spectra from a set of five different electrodes.

\begin{tabular}{lllll}
\hline Interface process & $\begin{array}{l}\text { Equivalent circuit } \\
\text { notation }\end{array}$ & Equation for impedance & Fit parameters & Unit \\
\hline Solution resistance & $R_{s}$ & $R_{s}$ & 63 & $\mathrm{~m} \mathrm{~cm}^{2}$ \\
Coating capacitance & $C_{c}$ & $\frac{1}{\mathrm{j} \omega C_{c}}$ & 10 & $\mathrm{mF} \mathrm{cm}^{-2}$ \\
Pore resistance & $R_{p}$ & $R_{p}$ & 60 & $\mathrm{~m}^{2} \mathrm{~cm}^{2}$ \\
Interface capacitance & $Z_{C P E}$ & $\frac{1}{Q(j \omega)^{n}}$ & $Q=18 n=0.66$ & $\mathrm{mS} \mathrm{s}^{n} \mathrm{~cm}^{-2}$ \\
Charge transfer resistance & $R_{t}$ & $R_{t}$ & 0.21 & $\Omega \mathrm{cm}^{2}$ \\
Diffusion element & $Z_{T}$ & $\frac{1}{Y_{0} \sqrt{\mathrm{j} \omega}} \operatorname{coth}(B \sqrt{\mathrm{j} \omega})$ & $Y_{0}=0.12 B=0.05$ & $\mathrm{~S} \times \mathrm{s}^{n} \mathrm{~cm}^{-2} \mathrm{~s}^{0.5}$ \\
\hline
\end{tabular}

\subsection{Evaluation of noise components}

Having determined the physical properties of the coated electrodes, we next evaluated the noise components that occur during recording sessions. Recorded neural signals are affected by several noise sources, both of biological and nonbiological origin.

The non-biological sources: include the electronic noise due to the amplifier, the thermal noise and a number of noise sources associated with the double layer interface between solution and microelectrode surface [41, 42]. Since current fluctuations caused by shot noise can produce voltage fluctuations on an electrode due to non-zero impedance of the electrode, it is necessary to estimate the amplitude of such voltage fluctuations. The rms shot noise is proportional to the square root of the dc current in the microelectrodeamplifier input circuit [43]. Since in our case there was no polarizing voltage applied to the electrode, all dc current in the circuit was due to the amplifier input bias current. In modern MOSFET amplifiers, this current is of the order of $<10 \mathrm{pA}$, corresponding to the rms shot noise of $<0.2 \mu \mathrm{V}$ on $1 \mathrm{M} \Omega$ resistance in the $0.25-8 \mathrm{kHz}$ frequency band. Several other noise components were much larger in our setup, however, and thus shot noise was not considered in this paper.

The noise of biological origin includes: (a) the additional thermal noise component due to the presence of brain tissue, which increases the electrode impedance real part and (b) small neural signals, emitted by distant neurons/neuronal processes too far to be detected as single units and therefore considered as noise for most neural signal analyses [29, 44].

The contribution of all non-biological noise components was measured by recording signals from single microelectrodes immersed in a saline solution and then the contribution of biological noise was assessed by recordings in vivo.

To better explain our noise and neural recording results, here we present the analysis of our measurement/recording setup. Figure 4 (A) shows the equivalent circuit model of the measurement setup. $R_{s}$ being the electrical resistance of the saline solution and $V_{n}$ the neural signal generator, the actual recorded signal $V_{\text {rec }}$ is given by the voltage divider formed by the amplifier input impedance $Z_{\text {ampl }}$ and the electrode impedance $Z_{\text {elect }}$ while the neural signal generator is shunted by low solution resistance $R_{s}$. Consequently, as long as $Z_{\text {ampl }} \gg Z_{\text {elect }}$, the measurement setup can be modelled as ideal (except for its own intrinsic noise) with $V_{n} \cong V_{\text {rec }}$. The equivalent input circuit of the amplifier used in this work is depicted in figure 4(B). Figure 4(C) shows the impedance values across frequency of both microelectrodes and input amplifier. Since the amplifier input impedance is at least two orders of magnitude larger than the impedance of any electrode throughout all the tested frequency range (figure $4(\mathrm{C})$ ) it does not affect the measurements (figure 5(A)). Concerning the bandwidth, the recording system has an $8 \mathrm{kHz}$ six-pole low pass cut off frequency; thus all our analysis was limited to $\sim 8 \mathrm{kHz}$. The single pole $70 \mathrm{~Hz}$ high pass filter was digitally removed in order to perform data analysis at lower frequencies while an additional filtering by the headstage occurred below $1 \mathrm{~Hz}$ thus setting the lower limit of our 
(A)

\section{noise rms}

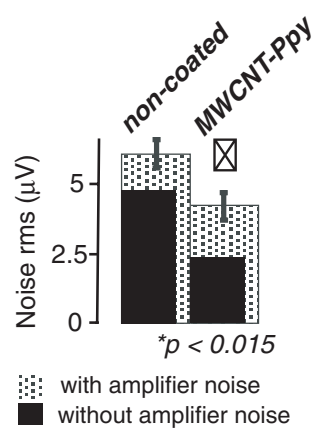

noise power

(C)

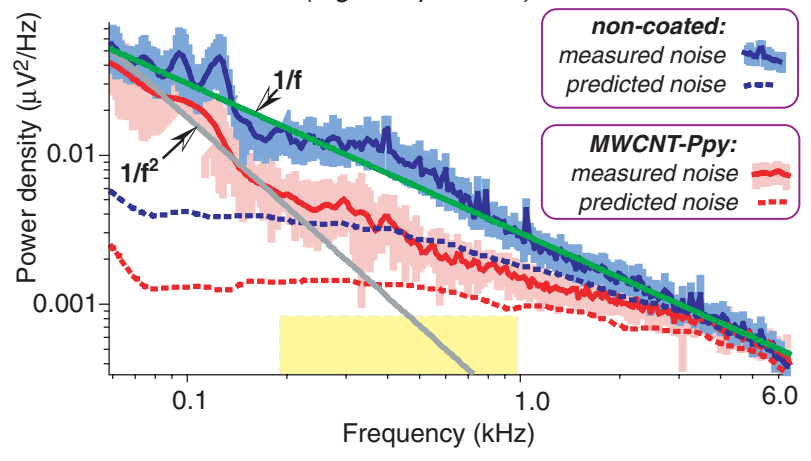

(B)

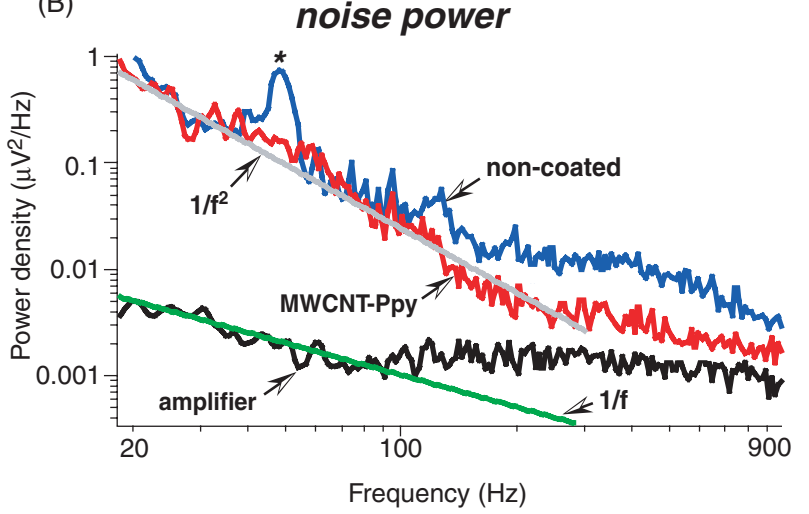

(D)

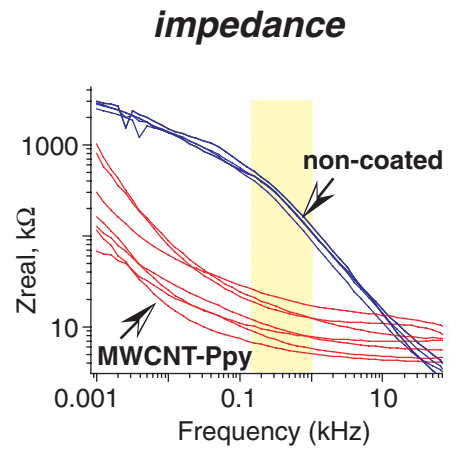

(E)

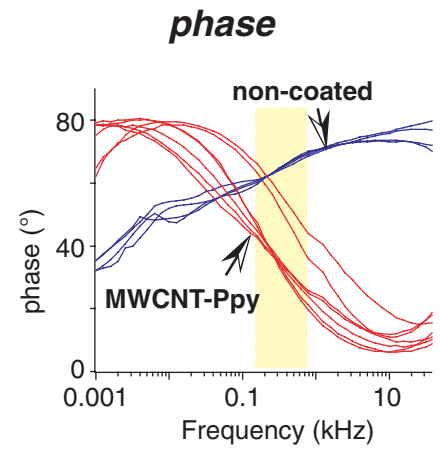

Figure 5. MWCNT-PPy coating reduces the background noise in the whole useful frequency range of the neural signal and in particular within the 200-1000 Hz frequency band. (A) Average rms noise is reduced in coated microelectrodes in saline solution tests. Light bars correspond to the total measured noise while dark bars inside correspond to the noise values obtained after subtraction of the electronic noise (see text for details). The frequency band is $0.25-8 \mathrm{kHz}$. Error bars correspond to the standard error of the mean. The difference between coated and non-coated microelectrodes is statistically significant for both values, with or without subtraction of the electronic noise ( $p<0.015$, one-tailed $t$-test). (B) The amplifier noise had negligible contribution to the overall noise below $100 \mathrm{~Hz}$. The noise SPDs of CNT-coated (red solid line) and non-coated (blue solid line) electrodes and short-circuited at the input Plexon amplifier (black solid line) are shown. In addition, grey and green lines representing $1 / f$ and $1 / f^{2}$ frequency dependence are shown for comparison. The asterisk indicates a peak of $50 \mathrm{~Hz}$ interference. (C) The average noise SPD graph obtained for non-coated and MWCNT-PPy microelectrodes in saline solution tests (continuous lines, accompanied in the right panel with a light shadow corresponding to the $95 \%$ confidence interval, for control and MWCNT-PPy-coated microelectrodes). The grey lines show the $1 / f$ function and a green line corresponds to $1 / f^{2}$ functions. The dashed lines are the predicted 'thermal + electronic' noise values for non-coated (blue) and MWCNT-PPy (red) microelectrodes (see the text for calculation details). The yellow area corresponds to the frequency range in which the noise SPD in the saline solution is significantly reduced after MWCNT-PPy coating. (D), (E) Impedance spectroscopy of non-coated and MWCNT-PPy-coated shows a significant decrease in the impedance real value (D) and a complete change in the phase frequency dependence (E). The light blue shaded area corresponds to the frequency range in which the background noise levels were reduced significantly after MWCNT-PPy coating.

analysis to $\sim 3 \mathrm{~Hz}$ (see also supplementary data available at stacks.iop.org/JNE/8/066013/mmedia).

The electronic noise in our system was measured by shorting the headstage inputs. The resultant combined amplifier plus headstage noise was $V_{\mathrm{rms}}^{\mathrm{amp}}=3.6 \mu \mathrm{V}_{\mathrm{rms}}$ in the $250-8000 \mathrm{~Hz}$ band, in good agreement with figures reported in Plexon datasheet [42]. In addition, we carried out two benchmark measurements with two resistors of 10 and $100 \mathrm{k} \Omega$, respectively. By doing this, we confirmed that the electronic noise values provided by the Plexon datasheet did not change for various input resistance values. The data of these measurements were used to build a 'thermal + electronic noise' predictor, on the basis of the electrode impedance, to estimate its contribution to the overall measured noise for each electrode (figure 5, see supplementary data available at stacks.iop.org/JNE/8/066013/mmedia for a detailed description).
It is well known that thermal noise is proportional to the square root of the real part of the electrode impedance $Z_{\text {real }}$ [20, 40]:

$$
V_{\mathrm{rms}}^{\mathrm{th}}=\sqrt{k_{b} T Z_{\text {real }} \Delta f},
$$

where $V_{\text {rms }}$ is the rms noise, $k_{b}$ is the Boltzmann constant, $T$ is the absolute temperature, $Z_{\text {real }}$ is the real part of the impedance and $\Delta f$ is the bandwidth over which the noise is measured. Assuming that the electronic noise is independent of other noise sources (our noise measurements with two different resistances are in agreement with such a notion), the predicted overall noise should be given by

$$
V_{\mathrm{rms}}^{\mathrm{tot}}=\sqrt{\left(V_{\mathrm{rms}}^{\mathrm{amp}}\right)^{2}+\left(V_{\mathrm{rms}}^{\mathrm{th}}\right)^{2}}
$$

For the average measured $Z_{\text {real }}$ of 56 and $8.3 \mathrm{k} \Omega$, the predicted integrated thermal noise in the $250-8000 \mathrm{~Hz}$ band was $\sim 2.3$ and $\sim 0.9 \mu \mathrm{V} \mathrm{rms}$ in pristine and in MWCNT-PPy microelectrodes correspondingly. 
Consequently, the predicted 'electronic + thermal' noise was $4.3 \pm 0.1 \mu \mathrm{V} \mathrm{rms}$ in non-coated and $3.7 \pm 0.05 \mu \mathrm{V} \mathrm{rms}$ in MWCNT-PPy-coated microelectrodes.

The actual noise values measured in a saline solution for pristine and coated microelectrodes in the $250-8000 \mathrm{~Hz}$ frequency band were $5.9 \pm 0.2$ and $4.2 \pm 0.3 \mu \mathrm{V} \mathrm{rms}$, respectively. These results have shown us that the MWCNTPPy coating reduced significantly the measured overall noise by $\sim 30 \%(p<0.015$; one-tailed $t$-test) (figure 5(A)) although these values were much higher than the overall predicted ones.

The additional non-electronic and non-thermal noise components, which should account for the difference between the measured and the predicted noise and could be calculated as the square root of the difference of the squares, was $\sim 3.9 \mu \mathrm{V} \mathrm{rms}$ in non-coated and $\sim 2 \mu \mathrm{V} \mathrm{rms}$ in MWCNTPPy microelectrodes. Thus, the average non-electronic and non-thermal noise component is larger than the thermal noise in both microelectrode groups.

A plot of the average noise spectral power density (SPD) obtained by testing the microelectrodes in a saline solution is shown in figure 5(B) (solid lines, blue for non-coated and red for MWCNT-PPy-coated microelectrode data, respectively). For both microelectrode types, the noise SPD increased at lower frequencies, particularly under $200 \mathrm{~Hz}$, suggesting the presence of a frequency-dependent noise. However, since it is well known that amplifiers show $1 / f$ noise at low frequencies, it is important to exclude the possibility that the amplifier noise shaped the electrode noise in the corresponding frequency band (for more details see supplementary data, figure S1 available at stacks.iop.org/JNE/8/066013/mmedia). To this end, based upon the measured microelectrode $Z_{\text {real }}$ and the measured combined electronic and thermal noise SPDs obtained on two resistors of 10 and $100 \mathrm{k} \Omega$, we estimated the total SPD of 'electronic + thermal' noise for non-coated and MWCNT-PPy-coated microelectrodes over the entire range of frequencies of interest.

Figure 5(B) shows that the combined effect of electronic and thermal noise could not account for the overall measured noise SPD. In particular, while below $70 \mathrm{~Hz}$ the Plexon amplifier noise SPD (black line, in this frequency range the thermal noise component contribution is small) scaled as $1 / f$ (green line), the electrode noise SPD for both kinds of electrodes at $70 \mathrm{~Hz}$ was $\sim 30$-fold higher than the amplifier noise and below $100 \mathrm{~Hz}$ all electrode noise SPDs increased faster with lower frequencies $\left(\sim 1 / f^{2}\right)$; for this reason we can conclude that the observed frequency dependence of the electrode noise below $100 \mathrm{~Hz}$ cannot be attributed to the amplifier noise. We therefore advanced the hypothesis that this non-electronic and non-thermal noise component is to be for the most part attributed to some frequency-dependent noise sources intrinsically related to the electrode. In the case of metal electrodes in solution, in particular, there are two types of frequency-dependent noise: the first has a $1 / f$ dependence on frequency $[41,45]$ and is generated by the charge carrier diffusion while the second scales with frequency as $1 / f^{2}$ and is generated by the charge carrier drift. Here we will refer to these two specific electrode interface noise components as ' $1 / f$ noise' and ' $1 / f^{2}$ noise', respectively.
While at frequencies below $100 \mathrm{~Hz}$, the measured noise SPD had a clear $1 / f^{2}$ dependence for both kinds of microelectrodes, the behaviour of the noise SPD at higher frequencies (above $100 \mathrm{~Hz}$, figure 5(C)) was quite different for the two types of microelectrodes. Although for all microelectrodes the measured overall noise (solid lines with shaded areas representing the $95 \%$ confidence intervals) is much higher than the predicted 'electronic + thermal' noise SPD (dashed lines), the frequency range in which this difference was significant is not the same for the two microelectrode types. In non-coated microelectrodes, up to $\sim 800 \mathrm{~Hz}$, the measured SPD is more than twofold larger than the predicted 'electronic + thermal' noise SPD. In MWCNT-PPy microelectrodes a more than twofold difference between the measured and the predicted SPDs is present only below $400 \mathrm{~Hz}$. In other words, from $\sim 400$ to $800 \mathrm{~Hz}$, the MWCNT-PPy microelectrodes showed noise SPD much closer to the predicted 'electronic + thermal' noise SPD than non-coated microelectrodes. In this frequency range in pristine microelectrodes the electrode interface $1 / f$ noise dominates as indicated by the overlay with a $1 / f$ function (green line in figure 5(C)). Meanwhile, in MWCNT-PPy microelectrodes the overall noise continues to decrease as $1 / f^{2}$ (grey line in figure $5(\mathrm{C})$ ) up to $\sim 250 \mathrm{~Hz}$ with no appreciable $1 / f$ noise contribution. Thanks to this impressive decrease in the $1 / f$ noise component in the frequency band between 400 and $800 \mathrm{~Hz}$, MWCNT-PPy coating results in a much larger overall noise SPD reduction (approximately threefold) than that predicted by the sum of the electronic and the thermal noise component SPDs (approximately twofold).

Above $1.5 \mathrm{kHz}$, there is no significant difference between the predicted and the measured noise SPDs. Moreover, above this frequency, probably due to the large electronic noise component and relatively small difference in the impedance real part (figure 5(D)), there is no significant difference between the non-coated and MWCNT-PPy microelectrode noise SPDs.

To conclude with the analysis of noise SPD measurements in the saline solution, we note that, notwithstanding the presence of an electronic noise component, the overall result is a significant reduction in the rms of total noise following MWCNT-PPy coating in the frequency band $250-8000 \mathrm{~Hz}$ ( $\sim 35 \%$, $t$-test, $p<0.05)$. In addition, noise reduction was particularly strong between $\sim 150$ and $1500 \mathrm{~Hz}$.

So far we compared non-biological noise spectra in noncoated and MWCNT-PPy-coated microelectrodes and found that $1 / f$ and $1 / f^{2}$ noise dominates at low neural signal frequencies. However, it is well known that the measured background noise is higher in the brain than in the saline solution, due to biological sources [20, 25, 29, 46]. First, the thermal noise component may increase because of the presence of brain tissue, so the resistive electrode impedance part, $Z_{\text {real }}$, could be larger than in the saline solution [40]. Second, a fraction of the neural activity recorded by an extracellular microelectrode, mostly generated by distant neurons $[15,29,47]$, cannot be differentiated from the overall background noise because of its small amplitude [29, 46, 48].

To discriminate between the contributions made to the background noise by these biological sources, we chose to 
(A) neural activity

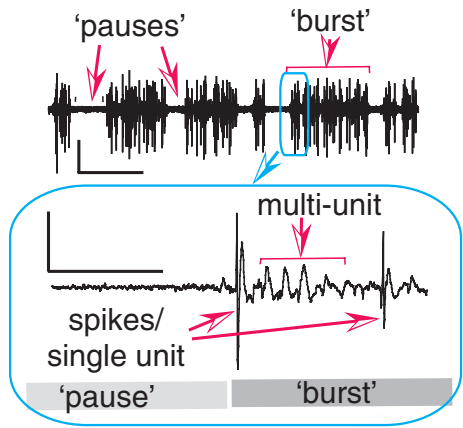

(B) tetrode tip

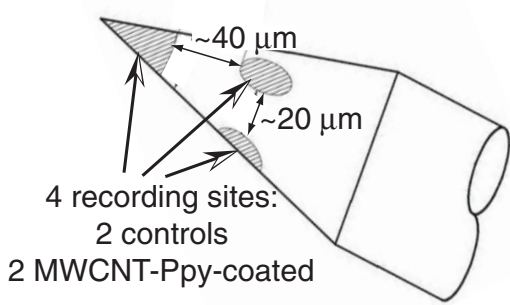

$$
\text { tetrode tip }
$$

(C)

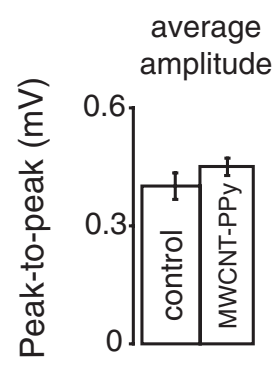

single-units

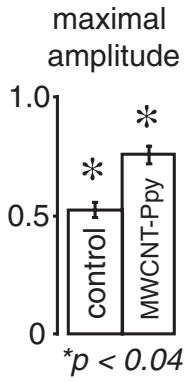

(E)

\section{local field potentials \\ 1-250 Hz, traces}

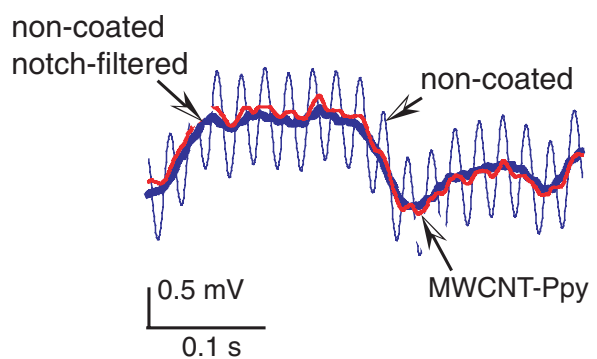

(D)

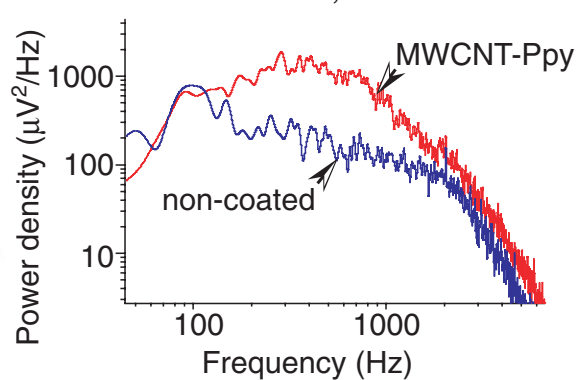

local field potentials 1-250 Hz, SPD

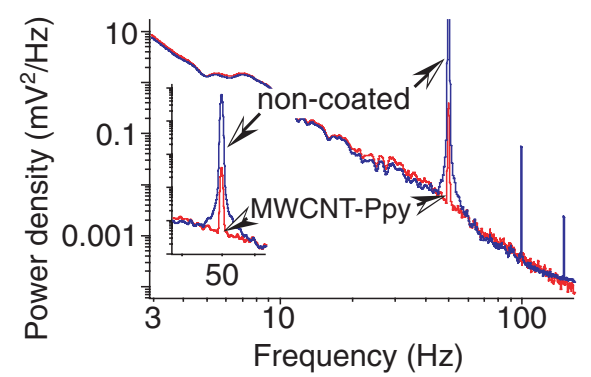

Figure 6. (A) A sample trace obtained in the deep somatosensory cortex of an anaesthetized rat (top). The scale bars correspond to $0.05 \mathrm{mV}$ and $0.1 \mathrm{~s}$. There are clearly distinct periods of low activity (pauses) and periods of high neuronal activity (bursts). An expanded fraction of the top trace at the border between a pause and a burst is shown below to demonstrate the presence of spikes (single units) and slower oscillations corresponding to a 'multiunit' signal. The scale bars for the bottom trace correspond to $0.05 \mathrm{mV}$ and $20 \mathrm{~ms}$. (B) The data presented in this figure (except for the 'multiunit' SPD graph) were obtained using tetrodes. The geometry of such a tetrode tip with four recording sites is shown. (C) The average amplitude of spikes in identified single units is unchanged by MWCNT-PPy coating (left columns) but the maximal spike amplitude is increased (right columns). (D) A plot of SPD obtained by subtraction of the 'pause' from 'burst' power spectrum. The significant increase of the SPD for the MWCNT-PPy-coated in the frequency range between 200-1000 Hz is evident. (E) A sample trace from coated (red trace) and non-coated (thin blue trace) recording sites of the same tetrode. The large $50 \mathrm{~Hz}$ component seen in the trace recorded by the non-coated site is dramatically reduced by MWCNT-PPy coating. Similar traces can be obtained using only notch filtering at $50 \mathrm{~Hz}$ (thick blue trace). (F) The SPD graph confirms this result. The inset shows at increased resolution the area around the 50 $\mathrm{Hz}$ interference. Note almost a third-order reduction in the $50 \mathrm{~Hz}$ component in the MWCNT-PPy-coated electrode trace. It must be underlined that in such a system the $50 \mathrm{~Hz}$ interference can easily drive the front-end amplifier to saturation and, in any case, multiple frequency notch filtering is necessary to recover the signal due to the higher harmonic contributions introduced by nonlinearity. The $50 \mathrm{~Hz}$ electromagnetic noise component rejection induced by microelectrode coating allows us to greatly reduce all those problems.

record from the deep layers of the primary somatosensory cortex (S1) in rats anaesthetized with Zoletil $\left(30 \mathrm{mg} \mathrm{kg}^{-1}\right)$ and Xylazine $\left(5 \mathrm{mg} \mathrm{kg}^{-1}\right)$, as under these conditions this part of the cortex switches between periods of high neural activity (bursts) and periods of much lower activity (pauses) [39], shown in figure 6(A). These periods of high and low activity are also synchronized for distant neurons, more than $1 \mathrm{~mm}$ apart, and during the 'pauses' the activity of distant neurons adds very little to the background noise [39]. Thus, 'pauses' provide us with a condition where the contribution of distant neurons is minimal while during 'bursts' we could evaluate the background noise levels when the activity of distant neurons is maximal. To estimate noise amplitude during bursts, in order to eliminate the effect of the presence of spikes [44], a median value was used (see section 2, subsection 2.6).

During 'pauses', on average, the overall noise was reduced from $6.9 \pm 0.2 \mu \mathrm{V} \mathrm{rms}$ in non-coated to $5.2 \pm$ $0.1 \mu \mathrm{V} \mathrm{rms}$ in MWCNT-PPy-coated microelectrodes $(p<$
0.0005 , one-tailed $t$-test), an $\sim 32 \%$ reduction. Following the subtraction of the electronic noise $(3.6 \mu \mathrm{V} \mathrm{rms}$, for the procedure see supplementary data available at stacks.iop.org/JNE/8/066013/mmedia), the background noise was $5.9 \pm 0.3 \mu \mathrm{V}$ and $3.7 \pm 0.3 \mu \mathrm{V} \mathrm{rms}(p<0.0005$, one-tailed $t$-test), respectively, corresponding to an $\sim 37 \%$ reduction. Thus, when the neuronal activity is low, the observed noise reduction is comparable to the data reported in the literature $(30-40 \%)[21,23]$ though smaller than in the saline solution test (55\%, see above). In contrast, during 'bursts', MWCNT-PPy coating did not reduce the background noise; actually there was an upward trend, from $11.2 \pm$ $3.0 \mu \mathrm{V} \mathrm{rms}$ in non-coated to $13.8 \pm 4.0 \mu \mathrm{V}$ rms in MWCNTPPy-coated microelectrodes $(p<0.2)$. This seemingly paradoxical increase in background noise can be explained by an increase of the signal component due to the distant neuronal activity. 


\subsection{Benefits of MWCNT-PPy coating for measuring neural activity}

Having investigated the noise sources both in the saline solution and in in vivo recording, we next performed the analysis of neural signals recorded from anaesthetized rats during acute sessions. Our aim was to compare the quality of neural signals recorded in vivo using either coated or uncoatedcontrol microelectrodes. We estimated the neural signal amplitude for single spikes ('single units', 500-3000 Hz, figures 6(A)-(C)), LFPs ( 1-250 Hz, figures 6(E) and (F)) and MUA ( $\sim 400-3000 \mathrm{~Hz}$, figures 6(A) and (D)). For these tests, we recorded from rat brain using quartz-platinum/tungsten tetrodes (figure 6(B), see section 2). In each of these tetrodes, two recording sites were coated with MWCNT-PPy while the other two were left untreated. As specified below, where possible, i.e. for MUA and LFP data, we made within-tetrode data comparisons. Even if sometimes it was not possible to have a direct, within-tetrode comparison (most single unit data), the ability to record from very closely spaced sites reduced data variability and permitted more accurate dataset comparisons. The impedance spectroscopy confirmed that the properties of these microelectrodes are very similar to the single wire platinum/tungsten microelectrodes. We made a total of six penetrations in two rats. In agreement with previous studies [20,21] we identified more neuronal units (see section 2, subsection 2.6) from MWCNT-PPy-coated recording sites than from non-coated ones (22 and 16 units respectively, data pooled from all tetrodes). As shown in figure $6(\mathrm{C})$, the coating increased the maximal spike amplitude from $0.52 \pm 0.03$ to $0.75 \pm 0.04 \mathrm{mV}$ ( $p<0.035$, one-tailed $t$-test, $n=17$, figure 6(C), data pooled from all tetrodes). These data are in good agreement with previous studies suggesting an increased sensitivity of CNT-coated microelectrodes $[20,21]$. However, despite the greater number of units recorded with the MWCNT-PPy-coated microelectrodes, the average amplitude of spikes was not significantly higher $(0.45 \pm 0.03$ versus $0.40 \pm 0.03 \mathrm{mV}, p<0.7$, data pooled from all tetrodes). These results can be explained by a skewed spike amplitude distribution, in which smaller spikes dominate affecting sorting and clustering analysis [46]. For such a distribution, the overall increase in spike amplitude will lead only to minor changes in the average amplitude if the threshold value used to detect spikes remains constant (see supplementary data available at stacks.iop.org/JNE/8/066013/mmedia for calculations). This is the case in our recordings, because the background noise during 'bursts', when most spikes were detected, was unchanged by MWCNT-PPy coating (see above) and therefore the spike detection threshold was set to a similar value in both cases. A larger background noise due to the presence of small, undetectable spikes from distant neurons (distant neuron noise) might explain why units were better separated in data from non-coated than coated electrodes. For matched amplitude units, the $L$ ratio was $0.006 \pm 0.002(n=9)$ and $0.08 \pm 0.02(n=11)$ in data from non-coated and MWCNTPpy microelectrodes respectively ( $p<0.03$, Mann-Whitney test). Similarly, isolation distance was $102 \pm 23(n=9)$ and $19.2 \pm 1.4(n=11)$ in data from non-coated and MWCNT-Ppy microelectrodes respectively ( $p<0.03$, Mann-Whitney test) [49]. Since the power spectra of distant neurons and detected spikes are similar, the distant neuron noise likely interferes with sorting more strongly than non-biological noise, which has spectral characteristics different from the single unit signal. We next estimated the contribution of MUA to the overall SPD by subtracting the recorded signal SPD obtained during 'bursts' from the one obtained during 'pauses'. We named this quantity the 'multiunit signal SPD'. Although few spikes were present in the bursts, their contribution to the signal power was minimal due to their brief duration $(<5 \%$ of all trace duration, figure 6(A)). The assumption that most of the SPD is due to multiunit signals was confirmed by the fact that the spike signal has a maximal power around $1 \mathrm{kHz}$ [7] while the multiunit signal SPD peaked well below $1 \mathrm{kHz}$ (figure 6(D)).

The SPD in figure 6(D) clearly shows us how coating with MWCNT-PPy enhanced such multiunit signal power between 150 and $\sim 2000 \mathrm{~Hz}$ with the maximal effect in a 200$1000 \mathrm{~Hz}$ band, where the signal power was increased up to a factor of 9 (two recording sites from the same tetrode were used). Since the total power in the MUA range reflects the total spike rate captured by the microelectrode [5] and smaller or more distant neurons generate signals of smaller amplitude $[1,15,46,47]$, the increase in MUA power observed with the coated microelectrodes suggests that they could capture spikes from neurons or axons that could not be detected by the uncoated microelectrodes because they were too far or too small.

It should be further stressed that using MWCNT-PPycoated microelectrodes, in the low frequency range, from which the LFPs are extracted, there was a remarkable reduction in the $50 \mathrm{~Hz}$ interference harmonics (the frequency of the European electric power network, figure 6(E)) and the power of this interference was reduced by more than 100 times (inset of figure $6(\mathrm{~F})$ lower panel, spectra from two sites of the same tetrode are presented), in good agreement with previous reports $[20,21]$. This is due to the very low MWCNT-PPy-coated microelectrode impedance. The SPD graph shows that there was little change at other frequencies except for the additional peaks of the $50 \mathrm{~Hz}$ interference harmonics in the non-coated microelectrode signal. The signal recorded from MWCNTPPy-coated microelectrodes was nearly identical to the one obtained from uncoated microelectrodes after notch filtering at $50 \mathrm{~Hz}$ (figure 6(E), top, traces are from two sites of the same tetrode). Since there was no change in non-biological noise for low frequencies under $150 \mathrm{~Hz}$ (figure 5(B)), after notch filtering of the $50 \mathrm{~Hz}$ interference, there was little change in the SNR in the LFP frequency range. There was, however, a very beneficial outcome of MWCNT-PPy coating for LFP studies: the decrease of $50 \mathrm{~Hz}$ noise obtained by coating the electrodes. This is important from a neurophysiological perspective for at least two reasons. First, it avoids the risk of amplifier saturation during LFP recordings. Second, the power line frequency of $50 \mathrm{~Hz}$ is a frequency in the middle of the so-called 'gamma range' which is thought to reflect a number of important brain functions $[50,51]$. Our results suggest that the coated microelectrodes offer considerable advantages for studying brain computations in the gamma frequency range. 
Noise reduction was particularly strong between $\sim 150$ and $1500 \mathrm{~Hz}$. The neurophysiological implications of these results is that the neural frequency range mostly benefitting from MWCNT-PPY coating is the one roughly covering the MUA band. A good SNR in this band is crucial both to evaluate (through multiunit activity) the overall amount of spiking activity around the tip of the electrode, and to extract well-isolated spiking activity of single neurons.

In summary, MWCNT-PPy coating increases the SNR of spiking signals and of gamma-range LFPs, without decreasing the SNR in lower frequency LFP bands.

\section{Conclusions}

We found that the most important improvements after MWCNT-PPy coating of microelectrodes are observed mostly in the frequency range from 150 to $1500 \mathrm{~Hz}$, mainly corresponding to the multiunit signal and, partially, to the high-frequency end of LFPs. In this frequency range, the nonbiological noise power is reduced up to threefold while the neural signal power is increased up to ninefold. Typically, the combination of lower noise and stronger neural signal produced approximately a fourfold SNR improvement in the middle of this frequency range. This result cannot be derived from the impedance spectroscopy, commonly the only method used to characterize the frequency-dependent noise features of extracellular microelectrodes [16, 20, 21].

We have been prompted to research in this direction by corrosion studies [45] and theoretical analyses [40] showing that, at low frequency, the so-called $1 / f$ noise contributes most to the overall noise and that the impedance measurements tell very little about $1 / f$ noise behaviour. Indeed, here we demonstrated that in a typical microelectrode, the thermal noise contribution to the overall noise is rather limited and became important only above $1-2 \mathrm{kHz}$, where only a small fraction of neural signal power is concentrated. In addition, we noted that there is a frequency range in which the neural signal amplitude is increased and this frequency range is similar to the one in which the noise SPD is decreased. Once again, a typical scheme used to account for neural signal attenuation that attributes most signal decrease either to a shunt capacitance or a high electrode resistance in sequence with the amplifier input resistance [7, 14] fails to explain the observed results. Indeed, the expected shunt capacitance for our immersion depths of $1-2 \mathrm{~mm}$ is less than $7 \mathrm{pF}$ corresponding to $>20 \mathrm{M} \Omega$ resistance at $1 \mathrm{kHz}$ [14]. Since the microelectrode resistance is always at least ten times smaller, no shunting is expected for any microelectrode. Similarly, we show that as at any given frequency the amplifier input impedance is several orders of magnitude higher than the impedance of both modified and non-modified electrodes, consequently, no voltage divider effect attenuating the neuronal signal amplitude should be expected. An equivalent circuit that was proposed in the past to model the electrode-solution interface impedance spectrum of electrodes modified by porous conducting polymers [31] allowed us to successfully reproduce the impedance spectra of our electrodes, but does not help to explain the increase in neuronal signal power following MWCNT-PPy coating that was observed. Taken together, these results suggest that the amplitude of the recorded neural signal cannot be predicted even with a relatively detailed lumped element equivalent circuit model. A more detailed approach, perhaps a microscopic/distributed conductance model, is likely to be needed for a more complete characterization of the propagation of neural signals at the electrode-solution interface. Finally, our results suggest that the presence of significant noise components that are neither thermal nor electronic can account for the absence of a direct relationship between the actual noise and the impedance spectra.

\section{Acknowledgment}

This work was supported by the BMI project of the Robotics, Brain and Cognitive Sciences Department, Italian Institute of Technology.

\section{References}

[1] Humphrey D R and Schmidt E M 1990 Extracellular single unit recording methods Neurophysiological Techniques: Applications to Neural System vol 15 (Clifton, NJ: Humana Press) pp 1-64

[2] Chorev E, Epsztein J, Houweling A R, Lee A K and Brecht M 2009 Electrophysiological recordings from behaving animals_-going beyond spikes Curr. Opin. Neurobiol. 19 513-9

[3] Abbott A 2009 Neuroscience: opening up brain surgery Nature 461 866-8

[4] Donoghue J P 2008 Bridging the brain to the world: a perspective on neural interface systems Neuron 60 511-21

[5] Logothetis N K 2003 The underpinnings of the BOLD functional magnetic resonance imaging signal J. Neurosci. 23 3963-71

[6] Buchwald J S and Grover F S 1970 Amplitudes of background fast activity characteristic of specific brain sites J. Neurophysiol. 33 148-59

[7] Fee M S, Mitra P P and Kleinfeld D 1996 Variability of extracellular spike waveforms of cortical neurons J. Neurophysiol. 76 3823-33

[8] Mehring C, Rickert J, Vaadia E, Cardoso de Oliveira S, Aertsen A and Rotter S 2003 Inference of hand movements from local field potentials in monkey motor cortex Nat. Neurosci. 6 1253-4

[9] Belitski A, Gretton A, Magri C, Murayama Y, Montemurro M A, Logothetis N K and Panzeri S 2008 Low-frequency local field potentials and spikes in primary visual cortex convey independent visual information J. Neurosci. 28 5696-709

[10] Pesaran B, Pezaris J S, Sahani M, Mitra P P and Andersen R A 2002 Temporal structure in neuronal activity during working memory in macaque parietal cortex Nat. Neurosci. 5 805-11

[11] Panzeri S, Brunel N, Logothetis N K and Kayser C 2010 Sensory neural codes using multiplexed temporal scales Trends Neurosci. 33 111-20

[12] Andersen R A, Burdick J W, Musallam S, Pesaran B and Cham J G 2004 Cognitive neural prosthetics Trends Cogn. Sci. 8 486-93

[13] Cogan S R 2008 Neural stimulation and recording electrodes Annu. Rev. Biomed. Eng. 10 275-309

[14] Robinson D A 1968 The electrical properties of metal microelectrodes Proc. IEEE 56 1065-71 
[15] Moffitt M A and McIntyre C C 2005 Model-based analysis of cortical recording with silicon microelectrodes Clin. Neurophysiol. 116 2240-50

[16] Loeb G E, Peck R A and Martyniuk J 1995 Toward the ultimate metal microelectrode $J$. Neurosci. Methods 63 175-83

[17] Cui X, Hetke J F, Wiler J A, Anderson D J and Martin D C 2001 Electrochemical deposition and characterization of conducting polymer polypyrrole/PSS on multichannel neural probes Sensors Actuators A 93 8-18

[18] Xiao Y, Cui X, Hancock J M, Bouguettaya M, Reynolds J R and Martin D C 2004 Electrochemical polymerization of poly(hydroxymethylated-3,4-ethylenedioxythiophene) (PEDOT-MeOH) on multichannel neural probes Sensors Actuators B 99 437-44

[19] Green R A, Williams C M, Lovell N H and Poole-Warren L A 2008 Novel neural interface for implant electrodes: improving electroactivity of polypyrrole through MWNT incorporation J. Mater. Sci., Mater. Med. 19 1625-9

[20] Ferguson J E, Boldt C and Redish A D 2009 Creating low-impedance tetrodes by electroplating with additives Sensors Actuators A 156 338-93

[21] Keefer E W, Botterman B R, Romero M I, Rossi A F and Gross G W 2008 Carbon nanotube coating improves neuronal recordings Nat. Nanotechnol. 3 434-9

[22] Yang Z, Zhao Q, Keefer E W and Liu W 2010 Noise characterization, modeling, and reduction for in vivo neural recording Adv. Neural Inf. Process. Syst. 22 2160-8

[23] Gabay T, Ben-David M, Kalifa I, Sorkin R, Abrams Z R, Ben-Jacob E and Hanein Y 2007 Electro-chemical and biological properties of carbon nanotube based multi-electrode arrays Nanotechnology 18035201

[24] Shoval A, Adams C, David-Pur M, Shein M, Hanein Y and Sernagor E 2009 Carbon nanotube electrodes for effective interfacing with retinal tissue Front. Neuroeng. 24

[25] Ludwig K A, Uram J D, Yang J, Martin D C and Kipke D R 2006 Chronic neural recordings using silicon microelectrode arrays electrochemically deposited with a poly(3,4-ethylenedioxythiophene) (PEDOT) film J. Neural Eng. 3 59-70

[26] Ansaldo A, Castagnola E, Maggiolini E, Fadiga L and Ricci D 2011 Superior electrochemical performance of carbon nanotube directly grown on sharp microelectrodes $A C S$ Nano 5 2206-14

[27] Yamato H, Ohwa M and Wernet W 1995 Stability of polypyrrole and poly(3,4-ethylenedioxythiophene) for biosensor application J. Electroanal. Chem. 397 163-70

[28] Buzsáki G 2006 Rhythms of the Brain (New York: Oxford University Press)

[29] Harris K D, Henze D A, Csicsvari J, Hirase H and Buzsáki G 2000 Accuracy of tetrode spike separation as determined by simultaneous intracellular and extracellular measurements J. Neurophysiol. 84 401-14

[30] Castagnola E, Biso M and Ricci D 2009 Improvement of polypyrrole and carbon nanotube co-deposition techniques for high charge-transfer electrodes Phys. Status Solidi b $2462469-72$

[31] Abidian M R and Martin D C 2008 Experimental and theoretical characterization of implantable neural microelectrodes modified with conducting polymer nanotubes Biomaterials 29 1273-83

[32] Klugh H E 1986 Statistics: The Essentials for Research 3rd edn (New York: Wiley)

[33] Hall R E and Lindholm E P 1974 Organization of motor and somatosensory neocortex in the albino rat Brain Res. $6623-38$

[34] Chapin J K and Lin C S 1984 Mapping the body representation in the SI cortex of anesthetized and awake rats J. Comp. Neurol. 229 199-213

[35] Paxinos G and Watson C 2007 The Rat Brain in Stereotaxic Coordinates 6th edn (Amsterdam: Elsevier)

[36] Woolsey T A and Van der Loos H 1970 The structural organization of layer IV in the somatosensory region (SI) of mouse cerebral cortex Brain Res. 17 205-42

[37] Simons D J 1978 Response properties of vibrissa units in rat SI somatosensory neocortex J. Neurophysiol. 41 798-820

[38] Brumberg J C, Pinto D J and Simons D J 1999 Cortical columnar processing in the rat whisker-to-barrel system J. Neurophysiol. 82 1808-17

[39] Erchova I A, Lebedev M A and Diamond M E 2002 Somatosensory cortical neuronal population activity across states of anaesthesia Eur. J. Neurosci. 15 744-52

[40] Lempka S F, Johnson M D, Barnett D W, Moffitt M A, Otto K J, Kipke D R and McIntyre C C 2006 Optimization of microelectrode design for cortical recording based on thermal noise considerations Conf. Proc. IEEE Eng. Med. Biol. Soc. $13361-4$

[41] Hassibi A, Navid R, Dutton R W and Lee T H 2004 Comprehensive study of noise processes in electrode electrolyte interfaces J. Appl. Phys. 96 1074-82

[42] Plexon Technical specifications in http://www.plexon.com/ assets/pdf/plexon_o25_headstages_techspecs.pdf

[43] Callegaro L 2006 Unified derivation of Johnson and shot noise expressions Am. J. Phys. 74 438-40

[44] Quiroga R Q, Nadasdy Z and Ben-Shaul Y 2004 Unsupervised spike detection and sorting with wavelets and superparamagnetic clustering Neural Comput. 16 1661-87

[45] Hladky K and Dawson J L 1982 The measurement of corrosion using electrochemical $1 / f$ noise Corros. Sci. 22 231-7

[46] Cohen I and Miles R 2000 Contributions of intrinsic and synaptic activities to the generation of neuronal discharges in vitro hippocampus $J$. Physiol. 524 485-502

[47] Henze D A, Borhegyi Z, Csicsvari J, Mamiya A, Harris K D and Buzsáki G 2000 Intracellular features predicted by extracellular recordings in the hippocampus in vivo J. Neurophysiol. 84 390-400

[48] Obeid I and Wolf P D 2004 Evaluation of spike-detection algorithms for a brain-machine interface application IEEE Trans. Biomed. Eng. 51 905-11

[49] Schmitzer-Torbert N, Jackson J, Henze D, Harris K and Redish A D 2005 Quantitative measures of cluster quality for use in extracellular recordings Neurosci. 131 1-11

[50] Gray C M, Konig P, Engel A K and Singer W 1989 Oscillatory responses in cat visual cortex exhibit inter-columnar synchronization which reflects global stimulus properties Nature 338 334-7

[51] Fries P, Nikolic D and Singer W 2007 The gamma cycle Trends Neurosci. 30 309-16 